Space Plasmas in the Solar System, including Planetary Magnetospheres (D) Space Climate (D2.5)

\title{
NEAR 13.5-DAY PERIODICITY IN MUON DETECTOR DATA DURING LATE 2001 AND EARLY 2002
}

Lucas Vieira, lucasfisico@gmail.com

National Institute for Space Research-INPE-Brazil, Sao Jose Dos Campos, Brazil Alisson Dal Lago, dallago@dge.inpe.br

National Institute for Space Research (Brazil), Sao Jose Dos Campos - SP, Brazil

Nivaor R Rigozo, nivaor.rigozo@crs.inpe.br

National Institute for Space Research-INPE-Brazil, Santa Maria, Brazil

Marlos Rockenbach Da Silva, marlos@univap.br

Universidade do Vale do Paraíba - UNIVAP, Sao Jose Dos Campos, Brazil

Carlos Roberto Braga, crbraga@dge.inpe.br

National Institute for Space Research (Brazil), Sao Jose Dos Campos, Brazil

Adriano Petry, adriano.petry@crs.inpe.br

Instituto Nacional de Pesquisas Espaciais - INPE, Santa Maria, Brazil

Nelson Jorge Schuch, njschuch@lacesm.ufsm.br

Southern Regional Space Research Center - CRS/CCR/INPE - MCTI, Santa Maria - RS, Brazil

In this study we perform a continuous Morlet Wavelet transform method in time series of secondary cosmic rays and 1 AU interplanetary medium parameters for the interval from October 2001 to October 2002. The approximately 13.5-day periodicity was obtained during the late 2001, and it was remarkable for muon data. Even though some works have pointed out that the main activations of the 13.5-day recurrence in near-Earth solar wind are related, e.g., with the heliosheet crossings or to the occurrence at $1 \mathrm{AU}$ of two high speed streams approximately $180^{\circ}$ apart in solar longitude per solar rotation, we aim to show that the period of about half the solar rotation during the end months of 2001 present in muon time series was apparently due to the occurrence of non-recurrent interplanetary disturbances. The interconnections among successive Forbush decreases, recovery phases and gradual muon depressions (associated with corotating interaction regions) seem to play an important role in such 13.5-day periodicity. 